\title{
Hippocampal function: Working memory or cognitive mapping?
}

\author{
DAVID S. OLTON, JAMES T. BECKER, and GAIL E. HANDELMANN \\ The Johns Hopkins University, Baltimore, Maryland 21218
}

\begin{abstract}
The relative merits of two different descriptions of hippocampal function are evaluated in a series of experiments examining the performance of rats following lesions in the hippocampal system. Cognitive mapping and working memory are operationally defined, and experimental tasks are categorized as requiring one or both of these two functions. In test procedures that have working-memory components, rats with fimbria-fornix lesions performed at chance levels and showed no signs of recovery of function even after extended postoperative testing. In test procedures that did not have working-memory components, rats with fimbria-fornix lesions had, at most, a short-term impairment. Thus, the performance of rats with lesions was systematically influenced by the memory characteristics of the task. Altering the cognitive mapping characteristics of tasks had much less of an effect; an impairment in a task that required working memory was found, irrespective of whether rats did or did not use a mapping strategy, and only transitory impairments were found in tasks that did not require working memory, irrespective of whether rats did or did not use a cognitive mapping strategy. These results support the hypothesis that the memory characteristics of tasks are the major factors influencing the magnitude of the impairment exhibited by rats with damage to the hippocampal system.
\end{abstract}

The hippocampus has been implicated as a structure necessary for both normal memory and normal spatial abilities. Evaluating the relative merits of these two descriptions of hippocampal function has been difficult because the results of many experiments can be successfully explained by either interpretation. Consider, for example, a series of experiments testing rats on an elevated radial-arm maze (Olton, 1978a, 1979; Olton \& Collison, 1979; Olton, Collison, \& Werz, 1977; Olton \& Samuelson, 1976; Olton \& Schlosberg, 1978; Walker \& Olton, 1979a). Each of the arms of the maze extended from a central platform like spokes on a wheel. At the beginning of each test, one food pellet was placed at the end of each arm. A food-deprived rat was placed on the central platform and allowed to choose among the arms. The optimal strategy was to choose each arm once and not to repeat a choice to any arm, thus getting all the food pellets in the minimum number of choices.

Normal rats learned rapidly and performed well.

The authors thank the participants at the Williams conference for helpful comments on the ideas expressed here and J. Krach for typing the manuscript. Partial support was provided by Research Grant MH 24213 from the National Institute of Mental Health and by a Biomedical Sciences Support Grant from the Johns Hopkins University to D.S.O. G.E.H. was supported by a predoctoral fellowship from the National Science Foundation. The authors thank the following for assistance in testing the rats used in these studies: C. Anderson, J. Argerson, T. Cox, D. Davies, B. Feustle, C. Foley, W. Franklin, R. Margolies, M. O'Regan, B. Papas, A. Reno, L. Sparzo, J. Steadman, B. Wolf, and S. Wolfe. J. Millstein helped with the data analysis.
On an eight-arm maze, they took about 10 tests to make an average of 7 correct responses in the first eight choices, and about 30 tests to make an average of 7.9 correct responses in the first eight choices (Olton \& Samuelson, 1976). The rats used extramaze cues to identify and remember each of the arms (Olton \& Collison, 1979; Olton \& Samuelson, 1976; Walker \& Olton, 1979a), and transfer tests suggested that they learned the topological relationship among the relevant extramaze stimuli (Suzuki, Augerinos, \& Black, in press; Black, Augerinos, \& Suzuki, Note 1).

Damage to the hippocampal system severely impaired rats' choice accuracy on this maze. Complete bilateral destruction of any of the extrinsic connections of the hippocampus was followed by performance that was near chance and showed no signs of recovery of function (Becker, Walker, \& Olton, in press; Becker, Walker, Olton, \& O'Connell, 1978; Olton, 1978b; Olton, Walker, \& Gage, 1978; Olton \& Werz, 1978; Walker \& Olton, 1979b). Damage to some of the intrinsic connections of the hippocampus produced severe deficits as well (Handelmann, Coyle, \& Olton, 1978; Jarrard, 1978a, 1978b; Handelmann \& Olton, Note 2). In contrast, lesions of other brain areas had little behavioral effect, a dissociation found after bilateral destruction of the posterior neocortex overlying hippocampus, medial frontal cortex, sulcal frontal cortex, caudate nucleus, and amygdala (Becker, Walker, \& Olton, in press; Becker et al., 1978; Olton et al., 1978; unpublished observations). Thus, performance on 
the elevated radial arm maze was selectively sensitive to hippocampal damage-sensitive because marked changes in behavior occurred following hippocampal damage, selective because these impairments were not found following other types of brain damage.

We suggested that this impairment occurred because the rats were unable to solve the memory component of the task. Abe Black suggested that the impairment occurred because the rats were unable to solve the spatial component of the task. Both suggestions are reasonable, of course, because an inability to identify the spatial locations of the arms or an inability to remember which locations had been chosen could have produced the observed impairment. Indeed, the relative merits of the two interpretations cannot be determined with this particular procedure because it contained both spatial and memory characteristics. As a result of many debates and discussions, we designed a series of experiments to test the alternative viewpoints. We will present here the operational definitions of the spatial and memory hypotheses that grew out of these discussions and describe the subsequent studies in which the memory characteristics and the mapping characteristics were manipulated independently. Our goals are three: (1) to evaluate the predictive validity of these two views of hippocampal function; (2) to demonstrate how a lesions analysis, in conjunction with appropriately designed experimental procedures, can be used to provide information about the functional organization of the brain; and (3) to acknowledge Abe Black's contributions to these experiments-both his concrete suggestions and his general ability to take abstract hypotheses and turn them into concrete experiments.

The rationales for both the spatial interpretation (Black, Nadel, \& O'Keefe, 1977; Nadel \& O'Keefe, 1974; Nadel, O'Keefe, \& Black, 1975; O'Keefe, 1976; O'Keefe \& Black, 1978; O'Keefe \& Conway, 1978; O’Keefe \& Dostrovsky, 1971; O’Keefe \& Nadel, 1978; O'Keefe, Nadel, Keightly, \& Kill, 1975) and the memory interpretation (Olton \& Papas, 1979; Olton, Becker, \& Handelmann, 1979) have been presented in detail elsewhere and will not be repeated here. Thus, the emphasis here will be on the experiments themselves. Furthermore, the experiments described here were designed specifically to address just these two hypotheses, and we will make no attempt to distinguish among other possible interpretations.

The discussion will focus on long-term behavioral impairments that follow complete bilateral destruction of the hippocampus proper or its major extrinsic connections through the fimbria-fornix or entorhinal area. Long-term impairments should be of greater significance than short-term ones (at least at the current stage of analysis) because they provide examples of behaviors that cannot be performed normally without the brain area in question. Furthermore, continued testing minimizes the possibility that the impairments are due to nonspecific changes in either the brain or behavior (Jarrard, 1975; Norman, 1973; Olton, 1978b; Olton, Becker, \& Handelmann, 1979). The emphasis on complete bilateral lesions follows from this interest in long-term impairments. Following partial hippocampal lesions, behavior often recovers to normal (Olton \& Papas, 1979). Such a recovery might be mediated by brain structures other than the hippocampus, in which case the hippocampus is not necessary for the recovered behavior. Alternatively, the recovery might be mediated by plastic changes within the hippocampus itself, suggesting that the hippocampus is necessary for the recovered behavior. Without additional experiments identifying the brain structures responsible for the recovery of function, conclusions about the role of the hippocampus in the recovered behavior cannot be made.

\section{Operational Definitions}

Working memory and reference memory. Workingmemory components of experimental procedures are those in which information on any single trial is useful only for that trial (Honig, 1978). Referencememory components of experimental procedures are those in which information on any single trial is useful for all the trials. An important point is that all behavioral tasks have a reference-memory component to them; the only question is whether or not there is also a working-memory component.

The distinction between working-memory and reference-memory components can be illustrated by considering a discrete-trial rewarded-alternation task in a T-maze. Each trial is composed of two runs through the maze. For the first run, one of the arms is blocked so that the rat is forced to go to the other arm. For the subsequent run, both arms are available and the rat can enter either one. On this free run, however, food is available only in the arm not entered on the first run of the trial. Following this trial, the rat is removed from the apparatus and returned to a holding cage to wait for the next trial. The arm that is available to the rat on the forced run varies from trial to trial. Consequently, the only way the rat can determine which arm has food on the free run of any given trial is to remember which arm was entered on the forced run of that trial; information about the arms entered on previous trials will not help to determine the arm that has food on the current trial.

The features of the reference-memory components of this task are: they remain constant from trial to trial; on the forced run of each trial, only one arm is available; food is found only in the food cups at the end of each arm; a run is terminated if the rat does not make a choice within 2 min; etc. The workingmemory component of this task is the alternation: 
the arm the rat enters on the forced run changes from trial to trial so that the arm that contains food on the free run also changes from trial to trial.

The elevated radial-arm maze experiment described in the introduction had a working-memory component to it (and may be considered as a variation of a rewarded alternation procedure). Within each test, on the first approach to an arm, the rat should go down that arm and get the food on that arm. On all subsequent approaches, the rat should not go down the arm because no food remains on it. Because the order in which the arms are chosen varies from test to test, the only way the rat can determine which arms still have food on them is to remember which arms have previously been chosen on that test. Because the elevated radial-arm maze task required working memory, the poor performance of rats following lesions of the hippocampal system might have arisen because they were unable to solve the workingmemory component of the task. We will call this explanation the "working-memory" hypothesis.

Cognitive mapping strategies and nonmapping strategies. An animal that uses a cognitive mapping strategy has learned the spatial relationships among the stimuli in his environment and is able to use this information to get from one place to another (Menzel, 1978; Olton, 1979). A prerequisite for the use of this strategy is an environment in which the discriminative stimuli maintain their topological relationship. Even in such an environment, cognitive mapping may not occur because there may be other ways to go successfully to the desired location. Determining whether an animal actually uses a cognitive mapping strategy in moving about the environment requires a transfer test. Three types of transfer tests have been developed. In the first, some, but not all, of the discriminative stimuli are removed. Use of a cognitive mapping strategy is indicated by successful performance with any subset of the stimuli remaining (O'Keefe \& Conway, 1978). In the second transfer test, all the discriminative stimuli remain but their topological relationship is changed. Cognitive mapping is indicated by a disruption of performance when the stimuli are interchanged, indicating that the topological relationship of the stimuli was an important dimension influencing the rat's choice behavior (Suzuki, Augerinos, \& Black, in press; Black, Augerinos, \& Suzuki, Note 1). In the third transfer test, the animal is first trained to go from several places in the environment to a goal, and is then given a test starting from a new location. Use of a cognitive mapping strategy is indicated by the animal choosing correctly on the very first transfer test and/or showing positive savings in learning to choose correctly from the new starting location (Olton, 1979; Tolman, Ritchie, \& Kalish, 1946).

In the following discussion, we will say that cognitive mapping (or a cognitive mapping strategy) is "permitted" when the stimuli in the environment maintain their topological relationship throughout testing, and that this strategy is "prevented" when these stimuli continuously alter their topological relationship. We will say that a cognitive mapping strategy is "used" when the environment permits cognitive mapping and the results of transfer tests are as described above.

In the elevated radial-arm maze task described in the introduction of this paper, the rats used extramaze stimuli to identify and remember each of the arms (Olton \& Collison, 1979; Olton \& Samuelson, 1976; Walker \& Olton, 1979a), and these stimuli maintained their topological relationship throughout testing. Thus, the procedure permitted a cognitive mapping strategy. Results from a transfer test suggest that the rats actually did use such a strategy (Suzuki, Augerinos, \& Black, in press; Black et al., Note 1). For those experiments, the maze was surrounded by a black curtain. Stimuli were placed on the curtain to provide extramaze stimuli identifying the location of each arm. One pellet of food was placed at the end of each arm, and the rat was rewarded for choosing each arm once. During training, the stimuli maintained a constant topological relationship on all tests. The actual position of the stimuli on the curtain changed from test to test (but remained constant within a test). After each rat learned to choose correctly, a transfer test was conducted. Each rat made three choices with the stimuli arranged as usual. The test was interrupted, the stimuli rotated or interchanged, and the rats reinforced for responding to the stimuli not chosen before. Rats in the rotation condition performed more accurately than rats in the interchange condition, suggesting that they had learned the topological relationship of the stimuli during initial training and were using a cognitive mapping strategy as defined above. ${ }^{1}$

Because the elevated radial-arm maze permitted a cognitive mapping strategy and normal rats used such a strategy, the poor performance of rats following hippocampal damage might have resulted from their inability to form a cognitive map. We will call this explanation of their impairment the "cognitive map" hypothesis.

\section{Experimental Design}

As described in the introduction, rats with damage to the hippocampal system performed no better than expected by chance when tested on an elevated radialarm maze. Because the task required working memory and because transfer tests suggested that normal rats used a cognitive mapping strategy, the deficit could have been due to either the memory or the mapping characteristics, and the results of that experiment cannot differentiate between these two explanations. Only other experiments, in which the memory and mapping characteristics are manipulated 
Table 1

\begin{tabular}{|c|c|c|}
\hline \multirow[b]{2}{*}{$\begin{array}{c}\text { Memory } \\
\text { Characteristics }\end{array}$} & \multicolumn{2}{|c|}{ Cognitive Mapping } \\
\hline & $\begin{array}{l}\text { Permitted } \\
\text { and Used }\end{array}$ & $\begin{array}{l}\text { Not Permitted } \\
\text { or Not Used }\end{array}$ \\
\hline $\begin{array}{l}\text { Working Memory } \\
\text { Required }\end{array}$ & & \\
\hline $\begin{array}{l}\text { Working Memory } \\
\text { Not Required }\end{array}$ & & \\
\hline
\end{tabular}

independently of each other, can evaluate the extent to which these two characteristics were responsible for the deficit. When this is done, a 2 by 2 table, as illustrated in Table 1, results. Experiments in the top row have a working-memory component as well as a reference-memory component; those in the bottom row have only a reference-memory component. Experiments in the left column are conducted in an environment that permits cognitive mapping and they include transfer tests indicating that the rats do, indeed, use a cognitive mapping strategy; those in the right column are either conducted in an environment that does not permit cognitive mapping strategies or, if it does, they include transfer tests indicating that the rats do not use these strategies.

The experiment described in the introduction is an example of a procedure from the upper left quadrant; working memory was required and cognitive mapping was both permitted and used. The predictions for the other quadrants are clear. If the deficit in the original elevated radial-arm maze experiment resulted from the working-memory requirements, then rats with damage to the hippocampal system should be impaired in all tasks that have a workingmemory component (top row), irrespective of their cognitive mapping characteristics, and should perform normally in all task that do not have a working-memory component (bottom row). If the deficit in the original experiment resulted from the cognitive mapping characteristics, then rats with damage to the hippocampal system should be impaired in all tasks in which cognitive mapping is used (left-hand column), irrespective of their memory characteristics, and should perform normally in all tasks in which cognitive mapping is either prevented or not used (right column). In the discussion that follows, this table will be used to categorize experiments on the basis of the definitions offered earlier and the available data.

\section{Cognitive Mapping Permitted, Working Memory Required in One Procedure But Not in a Second Procedure}

In the experiment described here (Olton \& Papas, 1979), the cognitive mapping characteristic was held constant while the memory requirement was changed, providing examples of procedures in the two left quadrants of Table 1 . The apparatus was a 17-arm radial maze. Like the other elevated mazes, it was placed in a room with many extramaze cues and each arm had only a short edge on it. At the beginning of each test, eight arms had one pellet of food placed at the end of each of them. The optimal strategy on this set of "baited" arms was to choose each of them once and only once. The other nine arms never had a food pellet at the end of them. The optimal strategy on this set of "unbaited" arms was never to choose them.

The baited arms form a working-memory component of this task. The first time the rat approaches a baited arm, he ought to choose it and obtain the food pellet at the end of it. On all subsequent approaches, he ought not to choose the arm because the food has been removed. Furthermore, the memory of the arms chosen on a previous test will not help the rat to determine whether or not a particular baited arm still has food on it and ought to be chosen on the current test. The unbaited arms form a reference memory component of this task. The correct response every time the rat approaches the arm is the same-he should not choose it. This constant stimulus-responsereinforcement relationship is, of course, the hallmark of reference-memory procedures. In short, the original 8-arm maze procedure has been incorporated into a 17 -arm maze in which all the remaining arms never have food on them. If the working-memory characteristic of the original radial-arm maze experiment was responsible for the deficit of rats with lesions in the hippocampal system, then rats with lesions in the present procedure should make errors by returning to baited arms from which they have already obtained the food (the working-memory component) but should not make errors by going to the unbaited arms (the reference-memory component). If, on the other hand, the cognitive mapping characteristic of the original radial-arm maze experiments was responsible for the deficit of rats with lesions in the hippocampal system, then rats with lesions in the present procedure should perform equivalently on both the baited and the unbaited arms because they have the same cognitive mapping characteristic.

Preoperatively, the rats required approximately 40 tests to reach the criterion of seven correct responses in the first eight choices. Following control operations, they continued to perform well. Following complete fimbria-fornix lesions, they performed poorly on both the baited and the unbaited arms for the first few tests. Performance on the unbaited arms rapidly recovered, while performance on the baited arms remained impaired. At the end of postoperative testing, performance on the unbaited arms was virtually perfect, but performance on the baited 
arms was near chance. Thus, on each test, the rats rarely made an error by choosing an unbaited arm but made many errors by repeating choices to the baited arms they had already visited. These results demonstrate that rats could distinguish between the set of baited and unbaited arms, remember which arms had food and which did not, and inhibit responses to the unbaited arms. Their only deficit was an inability to avoid returning to a baited arm once they had removed the food from it. Because this was a within-subjects, within-test dissociation, the results cannot be explained on the basis of elements that are common to performance on the set of baited and unbaited arms (such as the level of motivation, sensory or motor functions, etc.).

These results demonstrate that when the cognitive mapping characteristics of the original radial-arm maze procedure are held constant but the memory requirements are changed, there is a dissociation of performance following fimbria-fornix lesions. These findings are consistent with the hypothesis that the impairment of rats with fimbria-fornix lesions in the original elevated radial-arm maze task occurred as a result of the memory requirements of that task; they are inconsistent with the hypothesis that this impairment arose as a result of the cognitive mapping characteristic of that task. True, we did not conduct a transfer test to be certain that the rats used a cognitive mapping strategy. However, the apparatus and test environment were very similar to those used in the original experiments, and if the rats used a cognitive mapping strategy there, they could be expected to use a similar strategy here. Furthermore, any explanation of these results in terms of the cognitive mapping characteristics of the task would have to argue that the rats used two different strategies at the same time-a cognitive mapping strategy with the baited arms and a nonmapping strategy with the unbaited arms. Such a possibility is unlikely.

\section{Working Memory Required, Cognitive Mapping Prevented}

The experiment described here required working memory but prevented cognitive mapping (Olton \& Feustle, Note 3 ); it is an example of a procedure from the upper right quadrant of Table 1. A comparison of performance in this task with that in the original elevated radial-arm maze procedure provides an analysis complementary to the one just described; the working-memory characteristic of the tasks is held constant and the cognitive mapping characteristic is changed.

The apparatus was a four-arm radial maze constructed to enhance the salience of intramaze cues and suppress the salience of extramaze cues. Each arm had 10-cm-high walls and a top of cheesecloth which had been painted gray. During the experiment, the room lights were dim and a light was turned on in each arm so that the cheesecloth functioned as a one-way screen, allowing the experimenter to observe the rat but preventing the rat from seeing out of the maze. Distinctive tactile and visual cues were placed in each arm.

At the beginning of each test, one pellet of food was placed at the end of each arm. The rat was placed in the center compartment of the maze. All four guillotine doors were raised, allowing him to choose an arm. When he returned to the center compartment, the doors were lowered. While he was confined to the center compartment (which did not move), the arms were moved to their appropriate new positions. The doors were raised, the rat was allowed to make a new choice, and this procedure continued until each of the arms had been chosen once. Because the position of the arms was changed after every choice and the remaining food moved with the arms, the rat had to identify each arm on the basis of its intramaze stimuli and ignore its position in the room in order to choose correctly. One group of rats was trained in an interchange condition; after each choice, the arms were moved and their topological relationship was changed, preventing the formation of a cognitive map.

If, in the original elevated radial-arm maze task, the impairment of performance following lesions of the hippocampal system occurred because of the working-memory characteristic of the task, a similar impairment should be observed here because both tasks had a working-memory component. If that impairment occurred because of the cognitive mapping characteristic, then an impairment should not be found here because the intramaze task prevented the use of a cognitive mapping strategy.

Preoperatively, rats required about 50 tests to learn the interchange procedure to a criterion of a mean of 3.8 correct responses in the first four choices for 10 consecutive tests. Following surgery, the performance of rats with control operations remained at this level of accuracy. The performance of rats with fimbria-fornix lesions, however, fell to chance levels (an average of 2.8 correct responses in the first four choices) and showed no signs of recovering even after 60 postoperative tests.

These results demonstrate that the performance of rats with fimbria-fornix lesions in a task with a working-memory component was unaffected by changing the cognitive mapping characteristic of that task. The impairment found here, when the discriminative stimuli were intramaze cues with a changing topological relationship, was of the same magnitude as that found in the original experiments when the discriminative stimuli were extramaze cues with a constant topological relationship. These results suggest that the impairment in the original elevated 
radial-arm maze appeared because of the memory requirement of that task, and not because of its cognitive mapping characteristic.

\section{Working Memory Not Required, Cognitive Mapping Permitted and Used in One Procedure But Not in a Second Procedure}

The experiments described here (Becker \& Olton, 1980; Becker, Olton, Anderson, \& Margolies, 1979) compared performance in tasks which are examples of the two right quadrants of Table 1 . Both procedures required only reference memory. In one of them, mapping was permitted and transfer tests indicated that it was used. In the second, mapping was prevented.

The floor of the test apparatus was $1.4 \mathrm{~m}^{2}$, and there was a wall, $35 \mathrm{~cm}$ high, placed $10 \mathrm{~cm}$ from the outside edge of the floor. The area inside the wall was called the "test arena," and the area outside the wall was called the "runway." Three doors, each $10 \times$ $12 \mathrm{~cm}$, were equally spaced along each of the four sides of the wall. Masonite covers at each door controlled access from the runway to the test arena. In the test arena were two three-dimensional objects which had different shapes and patterns of black and white stripes on their sides. In the center of the top of each object was a hole which served as a food cup.

Preoperatively, each rat was trained to run along the runway, enter through the open door into the test arena, jump on top of an object, push away a piece of cardboard which covered the food cup, and obtain the food pellet in the cup. They were then trained in one of two discriminations. For rats in the object discrimination procedure, the location of both objects changed from trial to trial and food was always located on the top of the same object. Consequently, the object with the food could be determined only by the stimulus characteristics of the objects themselves, and not by their location in the test arena or by their position with respect to the rat when he entered the test arena from the runway. For rats in the location discrimination procedure, the objects changed from trial to trial and food was always located on the top of the object in the same location. Consequently, the object with the food could be determined only by the location of the objects in the test arena and not by their stimulus characteristics or by their position with respect to the rat when he entered the test arena from the runway.

All rats were given 20 trials a day, 5 days a week. On each trial, only one of the doors between the runway and the test arena was open. The rats were trained in a series of stages and the criterion for completion of each stage was 18 correct responses in the 20 trials of a single day. The first stage was a " 3 -door test" in which the open door was always the center door on one of three sides. The second stage was a "9-door transfer test" in which the open door was one of the three doors on the same three sides. The third stage was a "4-door transfer test" in which the open door was the center door on one of the four sides. After criterion performance on this last transfer test, the rats received either control operations or lesions of the fimbria-fornix. They were then given a 3-door test, a 9-door transfer test, and a 12-door transfer test in which all the doors were used.

Preoperatively, the mean number of errors before rats reached criterion on the 3-door test was 14 for rats in the location discrimination and 26 for rats in the object discrimination. Performance on both transfer tests in both discrimination tasks was virtually perfect, indicating that rats in the location discrimination used a cognitive mapping strategy while those in the object discrimination did not. ${ }^{2}$ Because neither of these tasks required working memory, the working-memory hypothesis predicts that following fimbria-fornix lesions, rats should perform equivalently in both the object and location tasks; they should be able to perform both discriminations and should show positive transfer on the transfer tests. Because rats in the location discrimination used a cognitive mapping strategy while those in the object discrimination task could not use such a strategy, the cognitive mapping hypothesis predicts a dissociation of performance in the two tasks; following lesions, rats should be able to perform the object discrimination and show positive transfer on transfer tests in this task but be unable to perform the location discrimination and/or fail to show positive transfer on transfer tests in this task.

Postoperatively, in the location discrimination, rats receiving control operations showed excellent retention, taking only two errors to reach criterion performance on the 3-door test. These rats also performed well on the 12-door transfer tests, choosing correctly on more than $90 \%$ of the trials from the new doors. Rats with fimbria-fornix lesions did not perform as well. They took an average of 32 errors to reach criterion on the 3 -door test and chose correctly on $80 \%$ of the trials from the new doors on the 12-door transfer tests. This deficit was specific to the location discrimination. In the object discrimination, the number of trials to reach criterion performance on the 3-door test was four for rats with control operations and five for rats with fimbriafornix lesions. Both groups of rats chose correctly on more than $90 \%$ of the trials from the new doors on the 12-door transfer test.

The selective impairment of rats with fimbriafornix lesions in the retention of the location discrimination but not in the retention of the object discrimination does not support the idea that only the memory requirement of a task is important in determining whether or not a deficit will occur following damage to the hippocampal system. However, several points should be made about the results 
of this experiment, particularly in comparison with the results of the radial-arm maze experiments. First, the deficits observed here were transitory, while those observed in the working-memory components of the radial-arm maze procedures showed no evidence of recovering. Second, the rats with lesions in the location discrimination did perform more accurately than expected by chance on the transfer tests, suggesting that they had learned the location discrimination with a cognitive mapping strategy and used this strategy following the lesion. The ability of the rats with lesions to learn the task postoperatively and to perform better than expected by chance on the transfer tests is consistent with predictions of the workingmemory theory but not with those of the cognitive mapping theory. However, the transitory deficit in the retention of the location discrimination (but not of the object discrimination) suggests that there are variables other than the type of memory that may differentially influence the performance of normal rats and those with hippocampal damage.

\section{Conclusion}

The present experiments make two contributions. First, they provide an evaluation of the variables responsible for behavioral impairments following lesions in the hippocampal system. They suggest that the most important factor determining whether or not an impairment occurs is the memory requirement of the task, but that other characteristics of the task may play some role as well. Second, they provide an example of the way in which lesions can be combined with appropriately designed experiments to provide information about the functional organization of the brain. Particularly important in this analysis are operational definitions of the terms used and development of tasks that can separate the different components that are of interest.

In closing, we acknowledge Abe Black's contributions to the experiments described here. While he was living, he made valuable suggestions that influenced the experiments conducted here. Even now, his style (Black, 1975) of "strong inference" (Platt, 1964) still guides the research. Abe was excellent at taking ideas and turning them into experimental procedures with clear predictions, an approach to research we have tried to follow here. One of the lessons learned from Abe is that the best colleagues are also the harshest critics. Abe ranked high on this quality. He took the time to understand what we were trying to say and made an effort to point out the strengths and weaknesses of our position. As a result, he continually refined our ideas, made them more explicit, and forced us to develop ways of turning them into data. We offer our discussion of the relative merits of the working memory and cognitive map theories in the same spirit.

\section{REFERENCE NOTES}

1. Black, A. H., Augerinos, G., \& Suzuki, S. Stimulus control of behavior on the 8 arm maze. Paper presented at the annual meeting of the Canadian Psychological Association, Ottawa, Ontario, Canada, June 9, 1978.

2. Handelmann, G. E., \& Olton, D. S. Spatial memory following damage to $C A_{3}$ pyramidal cells by kainic acid: Impairment and recovery with preoperative training. Unpublished manuscript, 1979.

3. Olton, D. S., \& Feustle, W. Hippocampal function and nonspatial memory. Manuscript submitted for publication, 1979.

\section{REFERENCES}

Becker, J. T., \& Olton, D. S. Object discrimination by rats: The role of frontal and hippocampal systems in retention and reversal. Physiology \& Behavior, 1980, 24, 33-38.

Becker, J. T., Olton, D. S., Anderson, C. A., \& Margolies, R. S. Both object and location reversal are impaired after frontal or hippocampal system damage in rats. Society for Neuroscience Abstracts, 1979, 5, 113.

Becker, J. T., WAlker, J. A., \& Olton, D. S. Neuroanatomical bases of spatial memory. Brain Research, in press.

Becker, J. T., Walker, J. A., Olton, D. S., \& O'Connell, B. C. Neuroanatomical bases of short-term spatial memory in the rat. Society for Neuroscience Eighth Annual Meeting, 1978, 4, 73. (Abstract)

BLACK, A. H. Hippocampal electrical activity and behavior. In R. L. Isaacson \& K. H. Pribram (Eds.), Neurophysiology and behavior (Vol. 2) The hippocampus. New York: Plenum Press, 1975.

Black, A. H., Nadel, L., \& O'KeEfe, J. Hippocampal function in avoidance learning and punishment. Psychological Bulletin, 1977, 84, 1107-1129.

Handelmann, G. E., Coyle, J. T., \& Olton, D. S. Lesions of $\mathrm{CA}_{3}$ cells with kainic acid impairs spatial memory in rats. Society for Neuroscience, Eighth Annual Meeting, 1978, 4, 221. (Abstract)

Honig, W. K. Studies of working memory in the pigeon. In S. H. Hulse, W. K. Honig, \& H. Fowler (Eds.), Cognitive aspects of animal behavior. Hillsdale, N.J: Erlbaum, 1978.

JARRARD, L. E. Role of interference in retention by rats with hippocampal lesions. Journal of Comparative and Physiological Psychology, 1975, 89, 400-408.

JARRARD, L. E. Selective hippocampal lesions and spatial discrimination in the rat. Society for Neuroscience, Eighth Annual Meeting, 1978, 4, 222. (Abstract) (a)

JARRARD, L. E. Selective hippocampal lesions: Differential effects on performance by rats of a spatial task with preoperative versus post-operative training. Journal of Comparative and Physiological Psychology, 1978, 92, 1119-1127. (b)

Menzel, E. W. Cognitive mapping in chimpanzees. In S. H. Hulse, H. F. Fowler, \& W. K. Honig (Eds.), Cognitive aspects of animal behavior. Hillsdale, N.J: Erlbaum, 1978.

NADEL, L., \& O'KEEFE, J. The hippocampus in pieces and patches: An essay on modes of explanation in physiological psychology. In R. Bellairs \& E. G. Gray (Eds.), Essays on the nervous system. A Festschrift for Professor J. Z. Young. Oxford: Clarendon Press, 1974.

Nadel, L., O'Keefe, G., \& Black, A. Slam on the brakes: A critique of Altman, Brunner, and Bayer's response-inhibition model of hippocampal function. Behavioral Biology, 1975, 14, 151-162.

Norman, D. A. What have animal experiments taught us about human memory? In J. A. Deutsch (Ed.), The physiological basis of memory. New York: Academic Press, 1973.

O'KEEFE, J. Place units in the hippocampus of the freely moving rat. Experimental Neurology, 1976, 51, 78-109. 
O'Keefe, J., \& Black, A. H. Single unit and lesion experiments on the sensory inputs to the hippocampal cognitive map. In Functions of the septo-hippocampal system. Ciba Foundation Symposium 58. New York: Elsevier, 1978.

O'KeEFe, J., \& Conway, D. H. Hippocampal place units: Why they fire where they fire. Experimental Brain Research, 1978, 31, 573-590.

O'KeEfe, J., \& Dostrovsky, J. The hippocampus as a cognitive map. Preliminary evidence from unit activity in freely moving rats. Brain Research, 1971, 34, 171-175.

O'KeEFE, J., \& NADEL, L. The hippocampus as a cognitive map. Oxford: Oxford University Press, 1978.

O'Keefe, J., Nadel, L., Keightly, S., \& Kill, D. Fornix lesions selectively abolish place learning in the rat. Experimental Neurology, 1975, 48, 152-166.

Outon, D. S. Characteristics of spatial memory. In S. H. Hulse, W. K. Honig, \& H. Fowler (Eds.), Cognitive aspects of animal behavior. Hillsdale, N.J: Erlbaum, 1978. (a)

Outon, D. S. The function of septo-hippocampal connections in spatially organized behavior. In Functions of the septo-hippocampal system. Ciba Foundation Symposium 58. New York: Elsevier, 1978. (b)

Olton, D. S. Mazes, maps, and memory. American Psychologist, 1979, 34, 588-596.

Olton, D. S., Becker, J. T., \& HandelmanN, G. E. Hippocampus, space, and memory. The Behavioral and Brain Sciences, 1979, 2, 313-365.

Olton, D. S., \& Collison, C. Intramaze cues and "odor trails" fail to direct choice behavior on an elevated maze. Animal Learning \& Behavior, 1979, 7, 221-223.

Olton, D. S., Collison, C., \& Werz, M. A. Spatial memory and radial arm maze performance in rats. Learning and Motivation, 1977, 8, 289-314.

Olton, D. S., \& PAPAS, B. C. Spatial memory and hippocampal function. Neuropsychologia, 1979, 17, 669-682.

Olton, D. S., \& Samuelson, R. J. Remembrance of places passed. Spatial memory in rats. Journal of Experimental Psychology: Animal Behavior Processes, 1976, 2, 97-116.

Olton, D. S., \& Schlosberg, P. Food searching strategies in young rats: Win-shift predominates over win-stay. Journal of Comparative and Physiological Psychology, 1978, 92, 609-618.

Olton, D. S., Walker, J. A., \& Gage, F. H. Hippocampal connections and spatial discrimination. Brain Research, 1978, 139, 295-308.

Olton, D. S., \& Werz, M. A. Hippocampal function and behavior: Spatial discrimination and response inhibition. Physiology \& Behavior, 1978, 20, 597-605.
Platt, J. R. Strong inference. Science, 1964, 146, 347-353.

Suzuki, S., Augerinos, G., \& Black, A. H. Stimulus control of spatial behavior on the eight arm maze in rats. Learning and Motivation, in press.

Tolman, E. C., Ritchie, B. G., \& Kalish, D. Studies in spatial learning: 1. Orientation and the short cut. Journal of Experimental Psychology, 1946, 36, 13-24.

Walker, J. A., \& Olton, D. S. The roie of response and reward in spatial memory. Learning and Motivation, 1979, 10, 73-84. (a)

W Alker, J. A., \& Olton, D. S. Spatial memory deficit following fimbria-fornix lesions: Independent of time for stimulus processing. Physiology \& Behavior, 1979, 23, 11-15. (b)

\section{NOTES}

1. The results of this transfer test support the idea that the rats used the topological relationship among the extramaze stimuli to learn the task. However, there are two potential difficulties with this interpretation. First, the transfer tests were the first time the stimuli had been moved during a test. Thus, the rats may have treated the new arrangement of the stimuli as if it were a different environment and reset working memory; the decreased choice accuracy exhibited by rats in the rotation condition supports this interpretation. Second, the movement of the stimuli assumes that the rats used only a single stimulus to identify each arm. Because the rats were able to see many of the stimuli from the central platform, they may have identified each arm on the basis not only of the stimulus at the end, but also on the basis of the stimuli on either side. In such a case, changing the topological relationship of the stimuli would be equivalent to removing some of the components of a compound stimulus, and the decreased choice accuracy might have resulted from degrading the discriminative stimuli. (See also Olton, Becker, \& Handelmann, 1979.)

2. Other transfer tests are being conducted at the completion of testing to determine the discriminative stimuli used by the rats in the location discrimination to identify the location of the correct object. Rats that were tested with the maze rotated $90 \mathrm{deg}$ and the objects in the same location in the room performed almost perfectly. Thus, they must have identified the location of the correct object without reference to particular stimuli (knots, patterns in the wood, etc.) on the walls or floor of the test arena. Additional transfer tests will provide information about the relative importance of the extramaze cues surrounding the test arena as compared with the topological relationship between the objects and the walls of the test arena. 